\title{
Designed plasmonic nanocatalysts for the reduction of eosin Y: absorption and fluorescence study
}

Abha Komalam, Lekha Girija Muraleegharan, Suganthi Subburaj, Suji Suseela, Aswathy Babu and Sony George*

\begin{abstract}
In this work, we report a one-step green synthesis of gold nanoparticles (AuNPs) by microwave irradiation using nontoxic and biodegradable polysaccharide chitosan as a reducing and stabilizing agent. The interaction between gold nanoparticles with the amine group of chitosan was confirmed by Fourier transform infrared spectroscopy analysis, and the stability of the nanoparticle is ascertained by zeta potential measurements. Transmission electron microscopy photograph and dynamic light scattering measurements confirmed the average size of gold nanoparticles as $25 \mathrm{~nm}$. The ability of the synthesised gold nanoparticles as a catalyst for the reduction of eosin dye in the presence of $\mathrm{NaBH}_{4}$ was monitored by means of spectrofluorometry and spectrophotometry. It is found that the $\mathrm{NaBH}_{4}$-induced reduction of eosin is enhanced in the presence of AuNPs even without a catalyst. Time-resolved fluorescence decay studies also confirmed the reduction of eosin in the presence of AuNPs.
\end{abstract}

Keywords: Nanostructures, Catalytic properties, Optical properties

\section{Background}

There has been significant interest on metallic nanoparticles over the past several years because of their unique shape- and size-dependent physical and chemical properties that have been exploited for a surfeit of applications including optical sensing, catalysis, nanoscale electronics and biomedicine $[1,2]$. Among the various metallic nanostructures, gold with well-defined dimensions represents a particular class of nanomaterials to synthesize and study because of its extensive practical applications. The catalytic activity of nanoparticles which is attributed to the existence of enormous number of active sites available to the reactants [3] is well known. Of course, the efficacy of nanoparticle catalysts depends on various other factors such as morphology, average size distribution, porosity and phase composition [4,5].

The success of nanoparticles synthesis relies not only on the size- and shape-controlled distribution but also on the preparative routes. Much work has been devoted to the synthesis of AuNPs. In recent years, microwave chemistry has made a great impact and is a promising

\footnotetext{
* Correspondence: emailtosony@gmail.com

Department of Chemistry, University of Kerala, Kariavattom Campus, Kerala 695581, India
}

technique for the preparation of size-controlled metallic nanostructures. The greatest advantages of microwave heating is that it can heat a substance uniformly thus leading to a more homogenous nucleation and shorter crystallization time compared with those for conventional heating. This is beneficial to the formation of uniform and homogenous metal colloids [6].

Conventionally, synthetic techniques based on the reduction of gold ions with sodium citrate or sodium borohydride followed by surface modification of the produced particles with suitable capping ligands $[7,8]$ and organic solvents raised environmental concerns because of the toxic compounds used in the process. In order to minimize or eliminate pollution to the environment, many researches are now concentrating on biomolecules for the synthesis of AuNPs [9]. Raveendran et al. first developed the concept of completely green synthesis of gold nanoparticles using $\beta$-D-glucose as the reducing agent [10]. Mukherjee et al. reported a novel biological method for the synthesis of silver and gold nanoparticles using the fungus Verticillium [11]. In another study, Mandal et al. and Selvakannan et al. reported that amino acid such as aspartic acid [12] and tyrosine [13] can act as reducing agents for the synthesis of AuNPs.

\section{勿}


Chitosan is a natural polysaccharide obtained by the deacetylation of chitin. The biocompatibility, biodegradability, non-toxicity and absorption properties of chitosan have been explained for many applications. Wei and co-workers used it as a reducing agent to prepare gold nanoparticles by thermal and photochemical methods $[14,15]$.

Herein we present a simple, reliable one-pot synthesis of catalytically active gold nanoparticles stabilized by chitosan and their application in the reduction of eosin. A variety of interesting catalytic properties of gold nanoparticles in the reduction of different types of organic compounds and dyes have been reported. All of these studies have been limited to size-dependent catalytic properties and absorption studies. In this regard, we evaluate the gold nanoparticles-catalyzed reduction of eosin based on spectrofluorometric analysis and time-correlated single photon counting (TCSPC) method.

\section{Methods}

\section{Materials}

All the reagents were of analytical reagent grade. Hydrochloroauric acid was purchased from Priya Laboratories Pvt., Ltd, West Bengal, India. The reducing agents chitosan and sodium borohydride were purchased from Aldrich (Sigma-Aldrich Corporation, St. Louis, MO, USA). The organic dye eosin was purchased from SRL Ltd., Mumbai, India. Chitosan was dissolved in 1\% acetic acid solution before use.

\section{Synthesis of chitosan capped gold nanoparticles}

Chitosan solution was prepared by dissolving $50 \mathrm{mg}$ in $1 \%$ acetic acid solution. Due to the poor solubility of chitosan, the mixture was stirred and kept for $24 \mathrm{~h}$ until a clear solution was obtained.

In a typical synthesis of gold nanoparticles, a $300-\mu \mathrm{L}$ $0.005 \mathrm{M}$ of $\mathrm{HAuCl}_{4} 3 \mathrm{H}_{2} \mathrm{O}$ and $15 \mathrm{~mL}$ of chitosan $(0.5 \mathrm{~g} / \mathrm{L})$ were mixed in a beaker and the $\mathrm{pH}$ of the solution was adjusted to 5 . This solution was heated in a microwave oven at $600 \mathrm{~W}$ powers for $60 \mathrm{~s}$. After the reaction, the colour of the solution changed immediately from colourless to wine red, indicating the formation of AuNPs (Scheme 1).

\section{Catalytic studies of chitosan capped AuNPs for eosin reduction}

The reduction of dye eosin by $\mathrm{NaBH}_{4}$ was studied as a model reaction to probe the catalytic activity of chitosancapped AuNPs. The procedures were as follows: $200 \mu \mathrm{L}$ of $0.005 \mathrm{M}$ AuNPs was added to $3 \mathrm{~mL}$ of distilled water containing $0.001 \mathrm{M} 20 \mu \mathrm{L}$ eosin in a quartz cell. Immediately after the addition of $200 \mu \mathrm{L}$ of $0.5 \mathrm{M} \mathrm{NaBH}_{4}$, the absorption and emission spectra were recorded in 10-min intervals in the range of $400-700 \mathrm{~nm}$ at room temperature.

\section{Instruments and characterisation}

The optical properties of the particles were measured by a JASCO V 550 UV-Vis spectrophotometer. Fluorescence spectra of AuNPs were recorded on a JASCO FP750 spectrofluorometer. Fourier transform infrared spectroscopy (FT-IR) spectra were obtained from ALPHA FT-IR spectrometers of Bruker Analytical Instruments,. Surface charge measurements were performed with a Zeta potential Analyser (Zetasizer 300 HAS, Malvern Instruments, Worcestershire, UK). Transmission electron microscopy (TEM) was carried out on a Hitachi H-7650 microscope (Hitachi Ltd., Brisbane, CA, USA) at an accelerating voltage of $120 \mathrm{kV}$. The size of the nanoparticle was also analysed using dynamic light scattering (DLS) equipment. The time-resolved fluorescence experiments were performed using an IBM picoseconds single photon counting employing a 520-nm nano-LED excitation source and a Hamamatsu C 4878-02 microchannel plate detector (Hamamatsu Photonics, Hamamatsu, Shizuoka, Japan).

\section{Results and discussion}

Mechanism for the formation of chitosan-capped gold nanoparticles

Chitosan has been reported as a useful stabilizing polymer of nanoparticles of Ag, Au, Pd, Pt and Rh [16-18]. The basic method for synthesis of nanoparticles in chitosan is to disperse metal ion solution in the polymer and reduce them to a zero valent state [19].

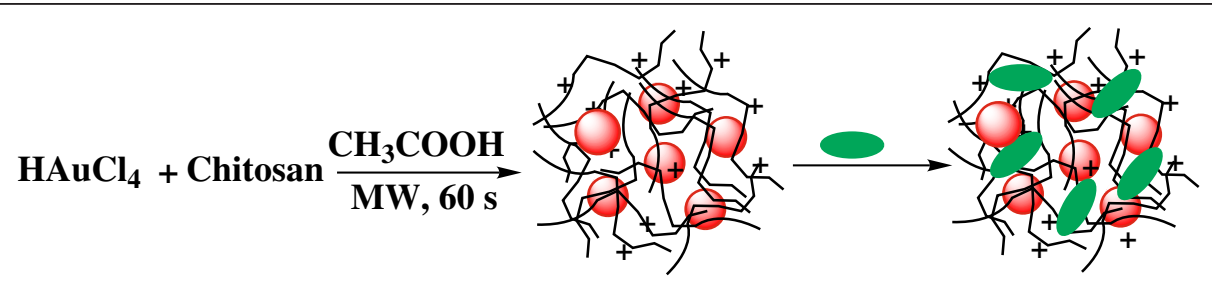

Gold Nanoparticles $+\overbrace{+}^{+}$Chitosan $\bigcirc$ Dye

Scheme 1 Schematic diagram showing the synthesis of chitosan-capped gold nanoparticles and subsequent adsorption of eosin dye. 
In acidic aqueous solution, the chitosan gets hydrolysed and the polymer chain is broken. One part of this polymer chain exists as an open-chain form. Once $\mathrm{Au}^{3+}$ ions were added in to the system, the $-\mathrm{CHO}$ group in the chain end would be oxidised to $-\mathrm{COOH}$ groups. Since oxidation reaction is irreversible, the dynamic equilibrium in the reaction would be broken and the reaction would proceed forwardly. Consequently, the chitosan chain is continually degraded and gold nanoparticles formed. A similar investigation was also done by Raveendran et al. [20,21]. The electrostatic attractive forces between amino groups in chitosan and metal salt ions in solution provide an effective driving force in the formation and stabilization of these metal nanoparticles [22].

In this work the synthesis was done in situ by the reduction of gold salt in the presence of chitosan under microwave heating for $60 \mathrm{~s}$ (Additional file 1). The chitosan acts as both reducing agent and capping agent. The concentration of chitosan required for the formation of gold nanoparticle was optimised to be $50 \mathrm{mg}$ (Additional file 2).

\section{Effect of $\mathrm{pH}$ on gold nanoparticles synthesis}

It is well known in literature that $\mathrm{pH}$ plays a predominant role in the synthesis of chitosan-capped gold nanoparticles [23,24]. In this work, the optimization was performed in the $\mathrm{pH}$ range of 3-10. Figure 1 compares the effect of $\mathrm{pH}$ on the surface plasmon resonance (SPR) band of AuNPs synthesised with $60 \mathrm{~s}$ of microwave

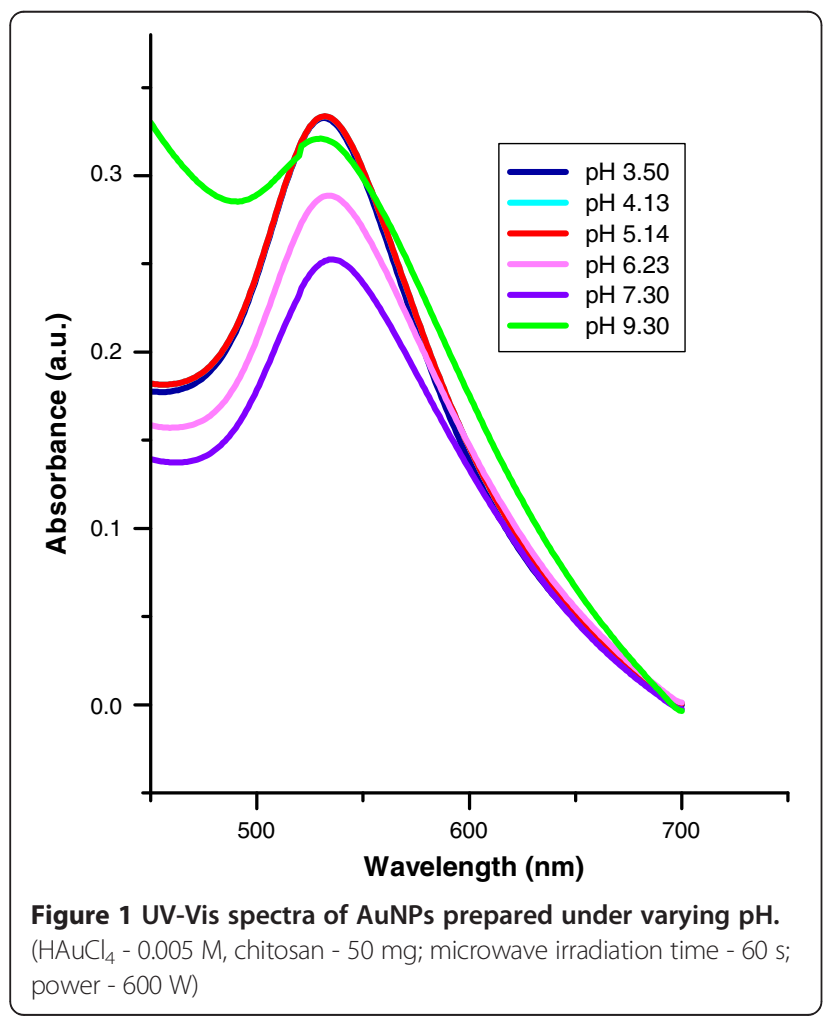

irradiation. The SPR peak intensity of AuNPs was almost same in the $\mathrm{pH}$ range of 3-5. When the $\mathrm{pH}$ increases to 5 , chitosan-induced aggregation takes place which leads to the surface plasmon coupling of nanoparticles. Due to this surface plasmon coupling, the SPR peak of AuNPs was slightly red-shifted. Surface plasmons decreases with decrease in the surface area are attributed to the particle aggregation which is clearly evident in the decline in absorbance value in the $\mathrm{pH}$ range from 5 to 7. At $\mathrm{pH}$ 9.3, particles are aggregated to such an extent that scattering increases the overall optical density. This is evident for the distinct change in the absorption curve from the typical Gaussian distribution pattern.

The above observation shows that the interaction of hydrochloroauric acid with chitosan and the increasing $\mathrm{pH}$ of medium via the increasing hydroxide ion concentration disfavour the hydrolyzation of $\mathrm{AuCl}_{4}^{-}$and, consequently, the formation of $\mathrm{Au}^{n+}$ [25], thus slowing down the reduction of $\mathrm{HAuCl}_{4}$ with chitosan. Therefore, the elevation of the $\mathrm{pH}$ of the medium did not facilitate the reduction of hydrochloroauric acid with chitosan. Decreasing the $\mathrm{pH}$ of the solution makes more protons available to protonate the amine group of chitosan, with the formation of a large number of cationic amines [26].

\section{TEM and DLS studies}

TEM as a powerful tool has been extensively used to investigate the morphologies and size distribution of the synthesized AuNPs. Figure 2 showed a typical TEM image of chitosan-capped AuNPs. The average particle size obtained is in the range of approximately $25 \mathrm{~nm}$.

DLS data of AuNPs synthesised at the $\mathrm{pH}$ of 5 was shown in Figure 3. The concentration of chitosan required

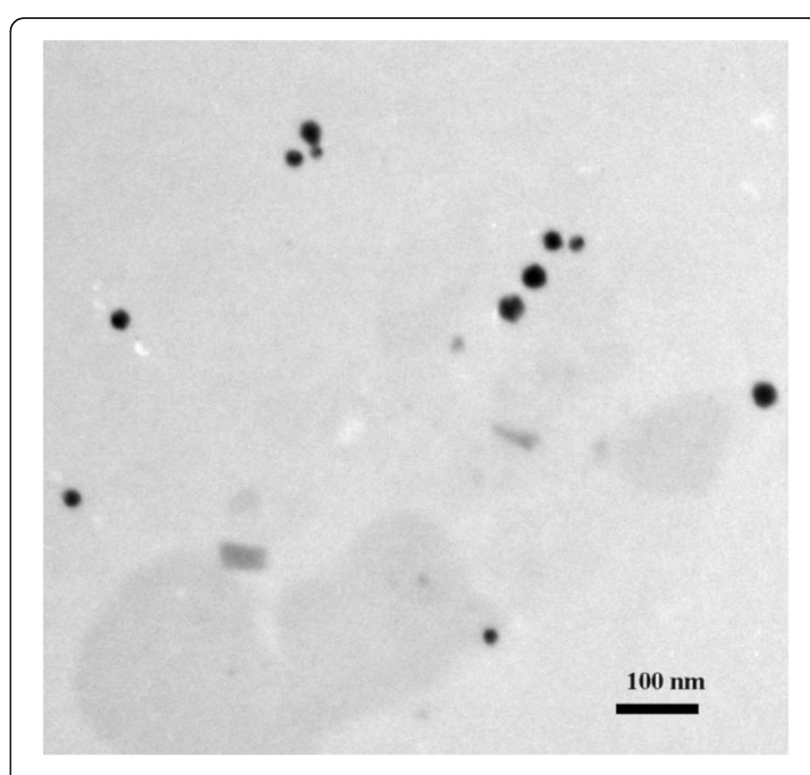

Figure 2 TEM image of chitosan capped Au nanoparticles. 


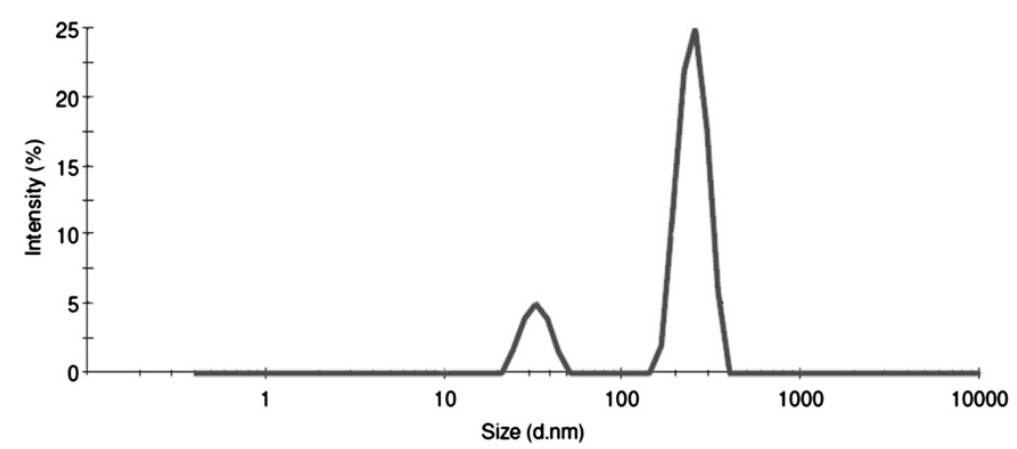

Figure 3 DLS size distribution graph of AuNPs synthesized using chitosan as reductant.

for the formation of gold nanoparticle was $0.5 \mathrm{mg} / \mathrm{mL}$. The DLS figure shows two size distribution peaks. The first peak centred on the hydrodynamic diameter of 33 $\mathrm{nm}$ attributes to the formation of chitosan-capped AuNPs. The later peak around $249 \mathrm{~nm}$ suggests the presence of unreacted chitosan in the solution, where they undergo self-cross-linking to produce chitosan polymer chain. In DLS analysis, hydrodynamic radius of the particle is measured, which is inversely proportional to the diffusion speed of the particles in the solution. The size of the hydration shell around each particle in the solution can affect the diffusion speed of the particles, subsequently the particle size. If the two particles have equal size, but one is more strongly coupled to the surrounding media, that particle will diffuse more slowly and register as larger. In our case, the particle size obtained from DLS analysis was much bigger than in its TEM photograph. This is because if the chitosan hydrogel shell on the nanoparticle swelled in aqueous solution, the water encapsulated in hydrogel shell would evaporate and nanoparticles shrunk remarkably in dry state for measurement with TEM.

\section{FT-IR spectral analysis}

FT-IR measurements were carried out to identify possible interaction sites between the gold salt and chitosan molecules, which are accountable for the reduction of the $\mathrm{Au}$ ions and stabilizing AuNPs.

Figure 4 (A) and (B) presents FT-IR spectra of pure chitosan and chitosan-capped gold AuNPs. It is clear from the figure that the $\mathrm{N}-\mathrm{H}$ bending vibration band at about $1,573.9 \mathrm{~cm}^{-1}$ were shifted to $1,546.9 \mathrm{~cm}^{-1}$ along with decrease in intensity [27]. This suggests the attachment of gold to nitrogen atoms, which reduces the vibration intensity of the N-H bond. It is notable that the chitosan-capped AuNPs showed a new band at $1,653 \mathrm{~cm}^{-1}$ which is attributed to the carbonyl stretch vibration in ketones, aldehyde and carboxylic group [28]. The presence of these peaks indicates that the reduction of the $\mathrm{Au}$ ions is coupled to the oxidation molecule and its hydrolyzates [29]. The appearance of the characteristic peak for the
- $\mathrm{COOH}$ groups supports the mechanism of degradations of the chitosan chain. Many previous reports have also confirmed that those compounds with hydroxyl groups such as glucose, ethylene glycol and polyols could be used as reducing agents for preparing the AuNPs [30,31].

\section{Zeta potential measurements}

Zeta potential is a crucial parameter for stability in aqueous nanosuspensions. For a physically stable nanosuspension solely stabilized by electrostatic repulsion, a zeta potential of $\pm 30 \mathrm{mV}$ is required as a minimum. Gold nanoparticles synthesised at $\mathrm{pH} 5$ with chitosan concentration of $0.5 \mathrm{mg} / \mathrm{mL}$ were taken for the zeta potential measurements. The chitosan-capped AuNPs had a zeta potential of $+49.4 \mathrm{mV}$, which suggested that the prepared nanoparticles were fairly stable.

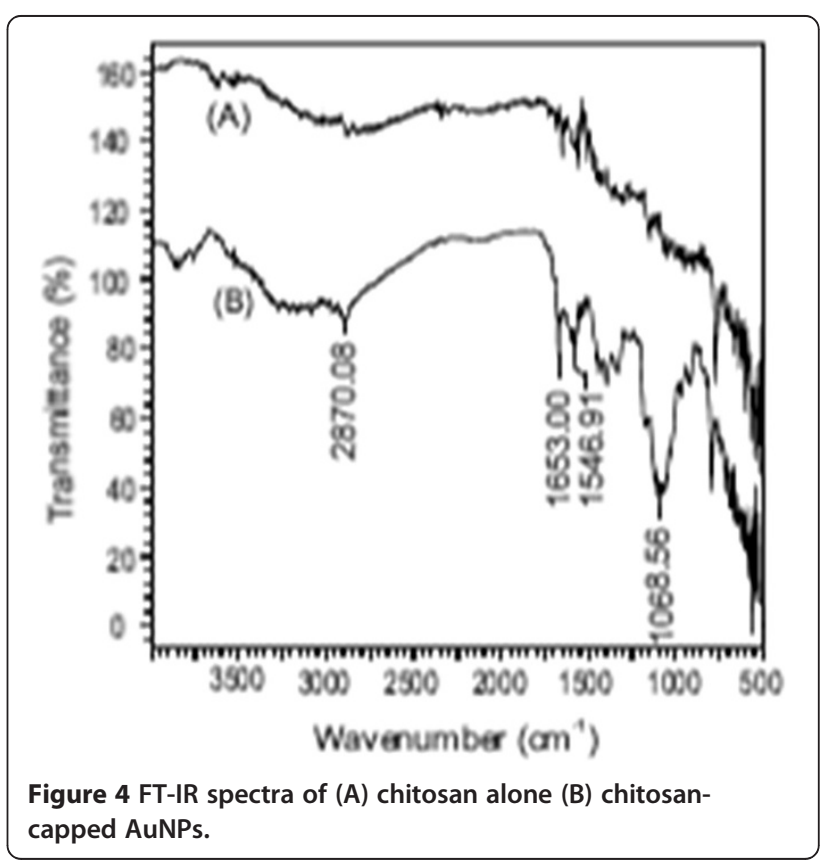




\section{Catalytic activity of AuNPs for the reduction of eosin}

Catalysis occurs only on the surface of metals; therefore, increasing the available surface area will greatly enhance the effectiveness of the catalyst. Decreasing the particle size will, in general, increase the catalytic activity; however, there is a critical size below which proves that further decreases will actually hinder the catalysis [32].

Metal nanoparticles help the electron relay from the donor to the accepter. The particles possess a large surface area which acts as a substrate for the electron transfer reaction. Just before the electron transfer reaction, both of the reactants are adsorbed on the metal particle. Subsequently, the reactant gains an electron and is reduced. Thus, the metal particles act as an efficient catalyst by being involved in the electron transfer process [33].

In this paper, the reduction of eosin by $\mathrm{NaBH}_{4}$ has been chosen as a bench-work reaction for monitoring the catalytic activity of AuNPs. Eosin is a wellstudied dye in the context of light energy conversion by sensitizing semiconductor nanoparticles in photoelectrochemical cells [34]. This reaction is chosen because it is a very 'gentle' reaction that is catalysed at room temperature.

\section{Absorption studies}

The AuNPs-catalysed reduction of eosin by $\mathrm{NaBH}_{4}$ was studied spectrophotometrically. For the reduction, $20 \mu \mathrm{L}$ of eosin was mixed with $3 \mathrm{~mL}$ of deionised water in a quartz cell. Then $200 \mu \mathrm{L}$ of chitosan-capped AuNPs solution was added and mixed by shaking. Finally, $200 \mu \mathrm{L}$ of $\mathrm{NaBH}_{4}$ was added to this solution. The progress of reduction was monitored in situ using a UV-Vis spectrophotometer.

Figure 5 shows the UV-Vis absorption spectra of the reduction reaction of eosin in the absence and presence of catalyst, chitosan-capped AuNPs. An aqueous solution of eosin shows an absorption maximum at $530 \mathrm{~nm}$. In the presence of AuNPs, eosin absorption spectra suffered a slight red shift in the band peak position, which indicated the adsorption of eosin on to the gold nanoparticles. Similar red shifts had been observed for eosin onto $\mathrm{ZnO}$ powder [35] and $\mathrm{TiO}_{2}$ [36]. Upon addition of $\mathrm{NaBH}_{4}$, the absorption band intensity decreased gradually, indicating the progress of the reduction of dye. But here, complete vanishing of absorption peak is not observed because the rate of reduction in water is extremely slow due to large kinetic barrier and low

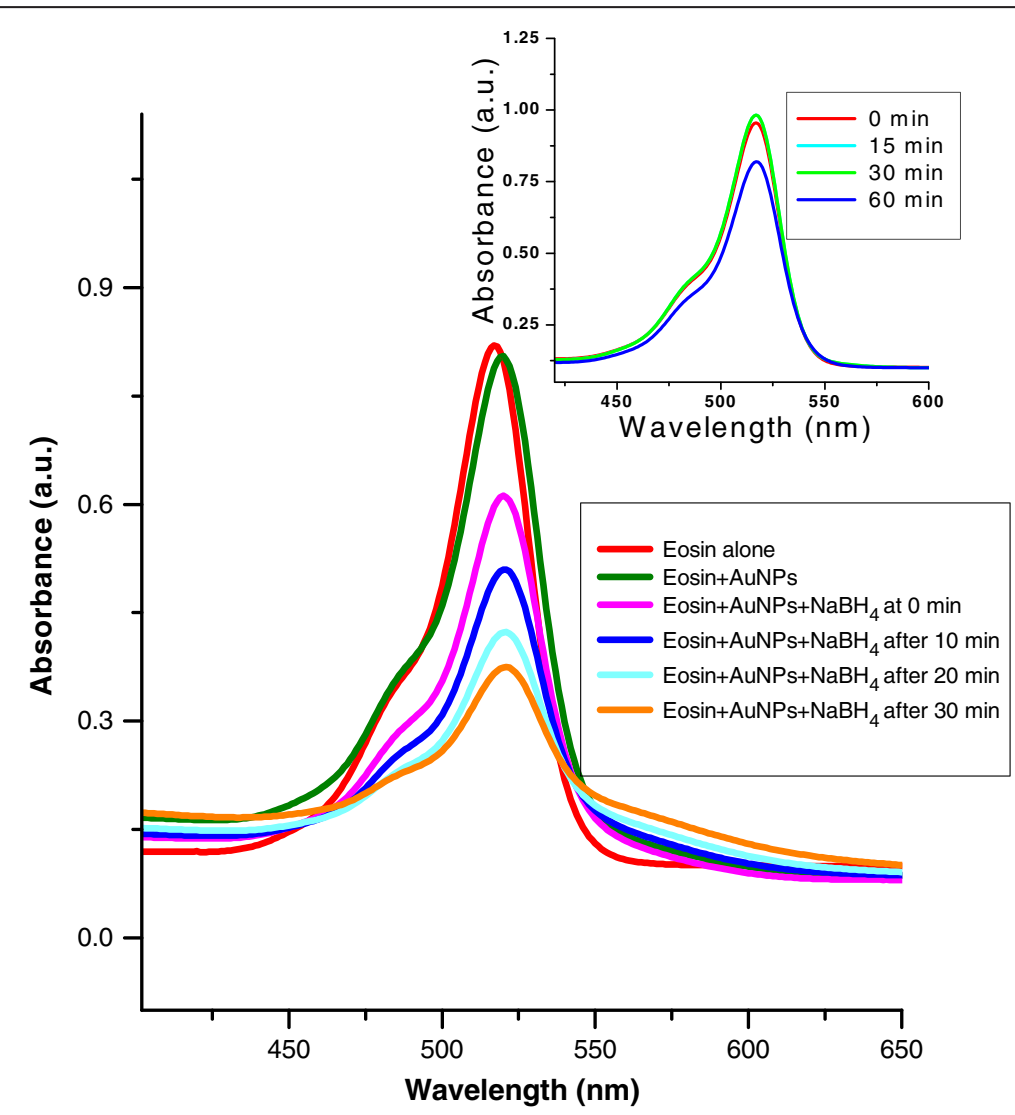

Figure $5 \mathrm{UV}$-Vis absorption spectra of the reduction of eosin by $\mathrm{NaBH}_{\mathbf{4}}$. UV-Vis absorption spectra of the reduction of eosin by $\mathrm{NaBH}_{4}$ in the presence of chitosan-capped Au nanoparticles as a catalyst. Inset: reduction of eosin by $\mathrm{NaBH}_{4}$ without AuNPs (HAuCl${ }_{4}-0.005 \mathrm{M}$, chitosan-50 mg, eosin-0.001 M,NaBH $4-0.5 \mathrm{M}$ ). 
encounter probability $[37,38]$. In the absence of AuNPs, there was almost no sign of reduction in the experimental time scale (shown as the inset of Figure 5).

On account of above observation, it is obvious that the AuNPs has acted as a catalyst to transfer the electron from $\mathrm{BH}_{4}^{-}$to eosin for their effective reduction. The mechanism is that the eosin dye is adsorbed on the AuNPs due to the electrostatic attractive force between positively charged chitosan-capped AuNPs and anionic dye eosin. Subsequently, the electron transferred from $\mathrm{BH}_{4}^{-}$to eosin for its reduction.

\section{Fluorescence studies}

The reduction of eosin in presence of AuNPs was also studied using florescence spectroscopy. For the fluorescence study, $200 \mu \mathrm{L}$ of $0.5 \mathrm{M} \mathrm{NaBH}_{4}$ was added to $3 \mathrm{~mL}$ of distilled water containing $0.001 \mathrm{M} 20 \mu \mathrm{L}$ eosin in a quartz cell. Immediately after the addition of AuNPs, emission spectra were recorded in 10-min intervals in the range of 400-700 $\mathrm{nm}$ at room temperature.

Figure 6 shows the fluorescence spectra for the reduction of eosin with the addition of AuNPs measured at 10-min interval during the reduction. The aqueous solution of eosin showed maximum emission peak at 570 nm. When AuNPs are added into eosin, a decrease in fluorescence intensity is observed. On adding $\mathrm{NaBH}_{4}$, the fluorescence further decreased gradually, and the reaction process was completed within $30 \mathrm{~min}$. However, the emission peak at $555 \mathrm{~nm}$ remained unaltered for a long duration when AuNPs were absent (inset of Figure 6), demonstrating that the reduction of eosin could not be realised alone with the reducing agent $\mathrm{NaBH}_{4}$.

\section{Time-resolved fluorescence decay studies}

Eosin in its monomeric form is a strongly fluorescing dye. In the presence of AuNPs the emission behaviour of the dye changes. Figure 7 (inset) shows the emission

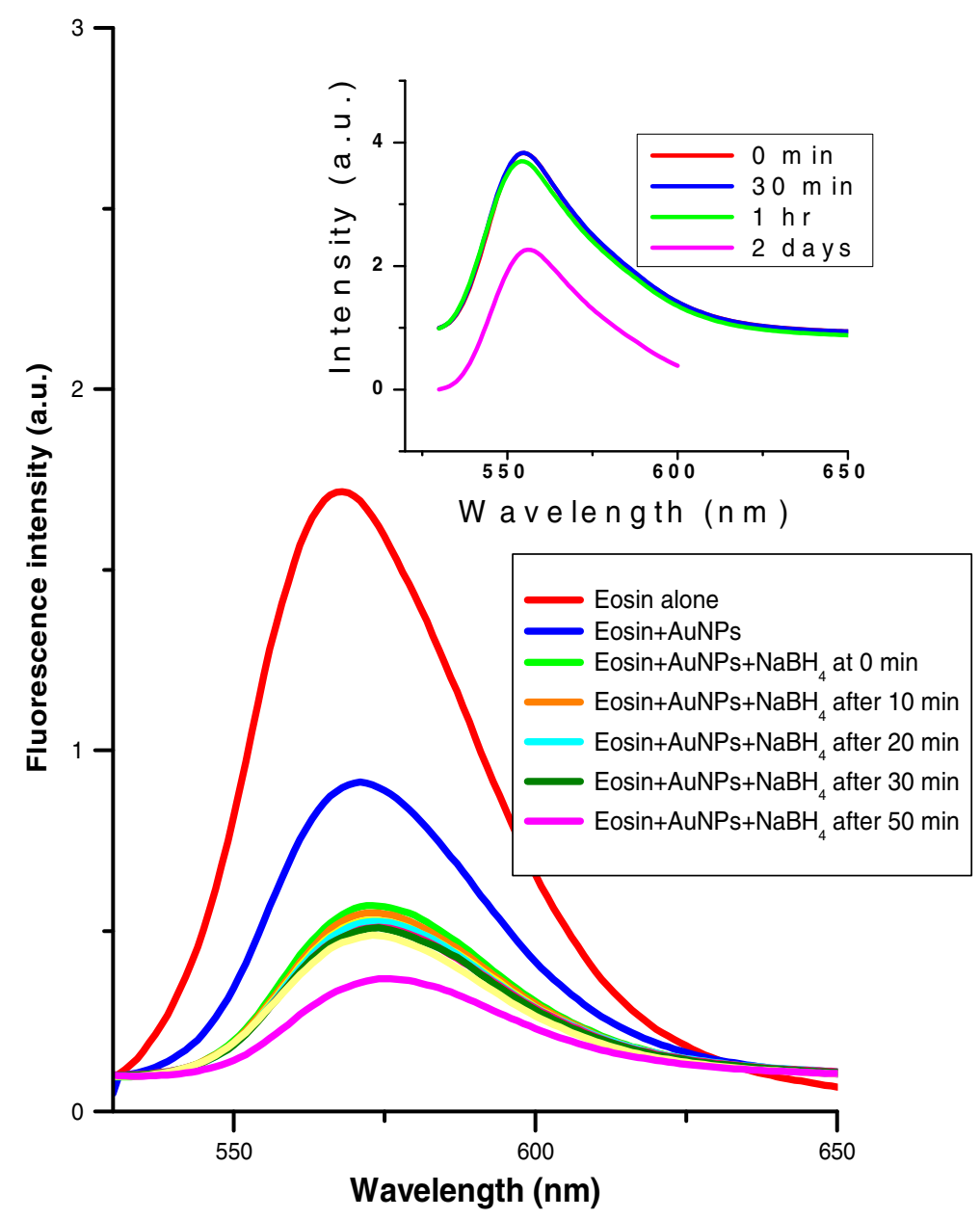

Figure 6 Fluorescence spectra of reduction of eosin by $\mathrm{NaBH}_{4}$ in the presence of chitosan-capped Au nanoparticles. Fluorescence spectra of reduction of eosin by $\mathrm{NaBH}_{4}$ in the presence of chitosan capped Au nanoparticles as catalyst. Inset: reduction of eosin by $\mathrm{NaBH}_{4}$ without AuNPs $\left(\mathrm{HAuCl}_{4}-0.005 \mathrm{M}\right.$, chitosan - $50 \mathrm{mg}$, eosin - $\left.0.001 \mathrm{M}, \mathrm{NaBH}_{4}-0.5 \mathrm{M}\right)$ 


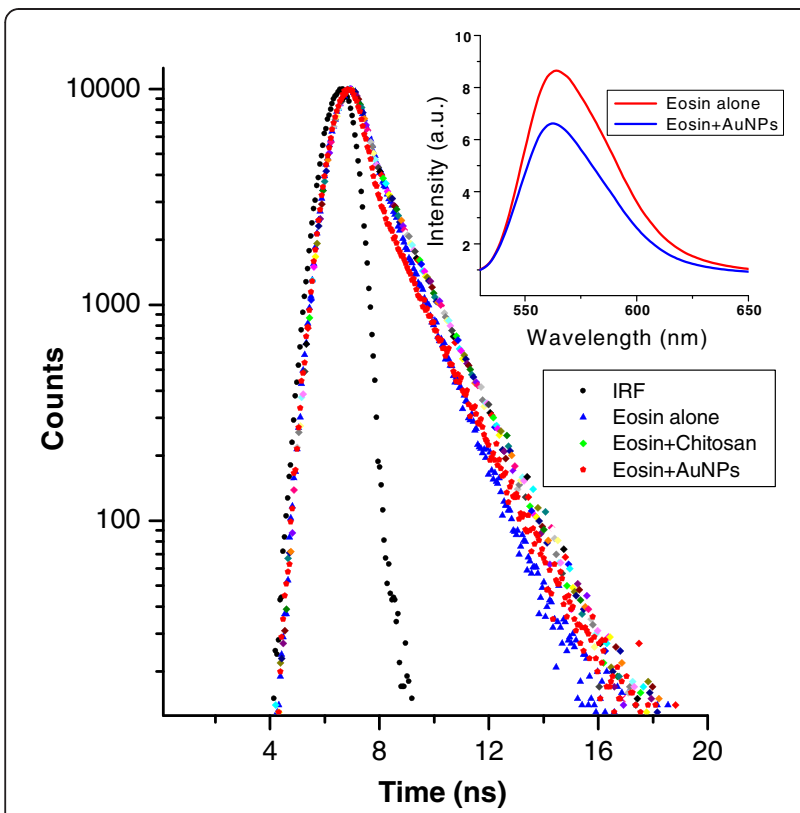

Figure 7 Time-resolved fluorescence decay spectra of eosin in presence and absence of AuNPs. Inset: quenching of dye fluorescence in presence of AuNPs alone $\left(\mathrm{HAuCl}_{4}-0.005 \mathrm{M}\right.$, chitosan - 50 mg, eosin - $0.001 \mathrm{M}$ )

spectra of eosin in the presence of AuNPs. Eosin exhibits an emission band with a maximum at $564 \mathrm{~nm}$. Addition of AuNPs to the eosin solution leads to decrease in emission intensity. The decrease in intensity can also be reasonably well correlated with the quenching of dye in presence of AuNPs in the solution. In order to ensure the decrease in fluorescence intensity is solely attributed to the catalytic activity rather than fluorescence quenching, the fluorescence lifetime studies were conducted.

The fluorescence lifetime is a relatively long process on the time scale of molecular events, and during this time, a high-energy fluorophore can undergo a great variety of transformations, ranging from electron redistribution and geometric alteration to reorganisation of the surrounding and chemical reactions [39]. A variety of fluorescence detection methods are available for lifetime measurement. The most widely used method is the TCSPC method.

The typical emission decay of eosin in the presence of AuNPs obtained from TCSPC method is shown in Figure 7 . The fluorescence decay studies were carried

Table 1 Time-resolved fluorescence decay profiles of eosin in the presence of AuNPs

\begin{tabular}{lccc}
\hline Samples & $\boldsymbol{\tau 1}(\mathbf{n s})(\%)$ & $\mathbf{\tau 2}(\mathbf{n s})(\%)$ & taverage (ns) \\
\hline Eosin in water & $0.51(61)$ & $1.6(39)$ & 0.7826 \\
Eosin + chitosan & $0.44(24)$ & $1.63(76)$ & 0.6931 \\
Eosin + chitosan + AuNPs & $0.40(43)$ & $1.63(57)$ & 0.6423 \\
\hline
\end{tabular}

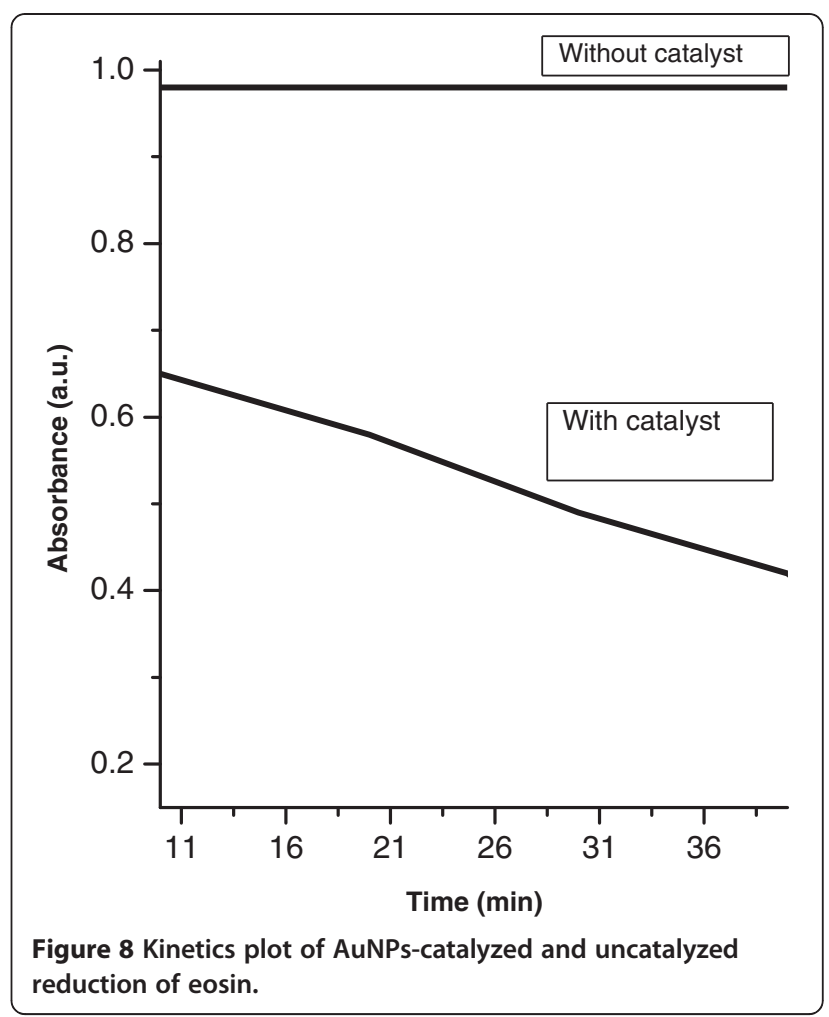

out with laser source at $560 \mathrm{~nm}$. From the figure, an instrument response function (prompt) reflects the distribution of photon from the excitation pulse in a nonfluorescent scattering media. In the presence of dye, the

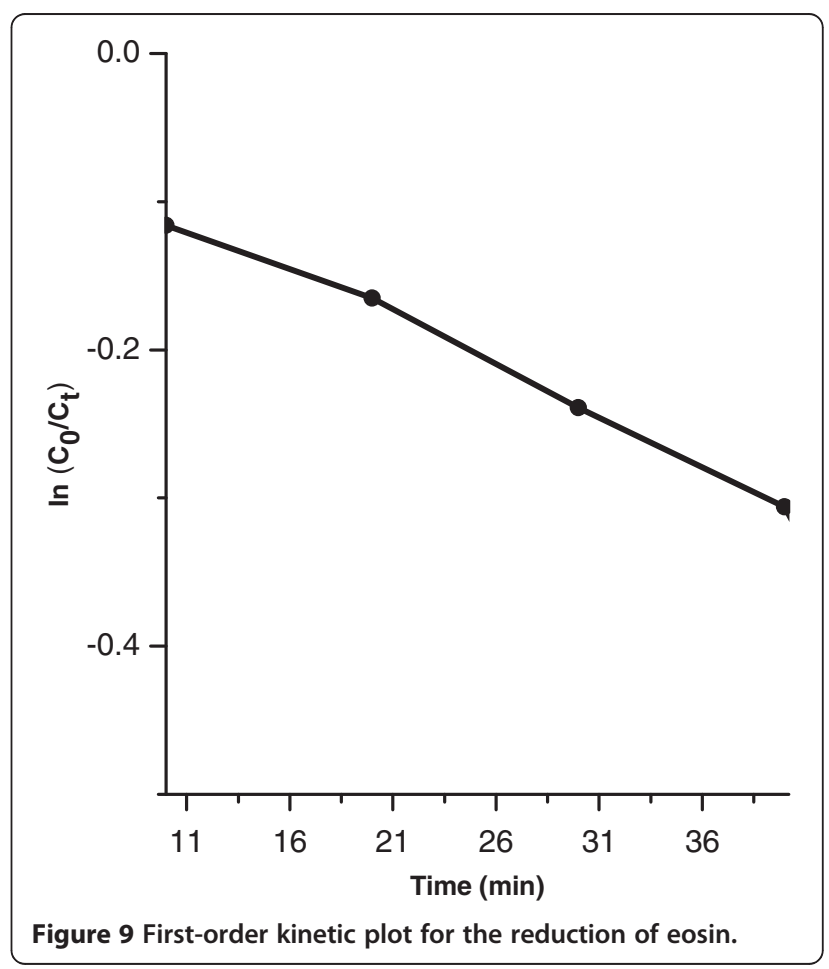


slope of the decay curve changes because of the existence of finite excited state. This decay curve remains unaltered in the presence of AuNPs. The time-resolved emission data that give a chi-square value of 1.0086 indicate accuracy of the fitting used, and the decay components were used to determine the average lifetimes.

Table 1 compared the average lifetime of pure eosin and eosin in the presence of AuNPs. The average lifetime of eosin-chitosan system in the absence and presence of AuNPs are 0.6931 and 0.6423 ns respectively. It is obtained as the inverse average lifetime equal to the weighted mean of the inverse time used for biexponential fitting. In the quenching process, the excited fluorescent molecules decrease their fluorescence lifetime via collision with other molecules, and the energy from the excited molecule is transferred to the quencher. The molecules are quenched as a result of multiple processes at the moment of collision, such as the formation of metastable complexes, resonance energy transfer, and electron transfer [39]. In our case, the average fluorescence lifetime differs by only $0.0508 \mathrm{~ns}$, and the decay curve remains almost unchanged. These studies ruled out the possibility of quenching of the fluorescence of dye in the presence of AuNPs.

On account of the above observation, we can conclude that quenching is not effective in eosin dye, and the decrease in intensity on the absorption and emission spectrum is mainly due to the reduction of eosin catalyzed by AuNPs.

\section{Kinetic studies}

Figure 8 shows the kinetic plot of the gold-catalyzed and the uncatalyzed reduction of eosin by $\mathrm{NaBH}_{4}$. From the figure it is clear that there is a fast decrease in the concentration of the eosin in presence of the AuNPs. In the absence of the catalyst, the kinetic plot remains as a straight line in the given time period. The particle concentrations in the solutions were calculated to be $6.67 \times$ $10^{-5}$ mol L ${ }^{-1}$ which is smaller than that of eosin. Since excess of $\mathrm{NaBH}_{4}$ was present in the reaction solution and the reduction of eosin by $\mathrm{NaBH}_{4}$ was negligible in the absence of AuNPs, the reaction could be considered as a pseudo-first order with respect to the concentration of eosin. Furthermore, as the absorbance of eosin is proportional to its concentration in the medium, the ratio of absorbance at time $t\left(A_{t}\right)$ to that at $t=0\left(A_{0}\right)$ i.e., $A_{t} /$ $A_{0}$ could be used as the ratio of concentration of eosin at time $t$ to that at $t_{0}$, i.e., $A_{t} / A_{0}=C_{t} / C_{0}$. As expected, a plot of In $\left(C_{t} / C_{0}\right)$ against time gives almost a straight line (Figure 9) and the rate constant can be obtained from the slope of the straight line. The rate constant $k$ was obtained as $8 \times 10^{-3} \mathrm{~s}^{-1}$.

\section{Conclusions}

In conclusion, we have developed a simple and facile method for the synthesis of AuNPs using chitosan as reducing and stabilizing agent. The resulting AuNPs displayed controllable structural and optical properties as demonstrated by absorption studies, fluorescence studies, DLS measurements and TEM observations. Results showed that the particles are mostly spherical in nature having an average size around $25 \mathrm{~nm}$. FT-IR analysis and zeta potential measurements confirm the coordination of the AuNPs to the amino group of chitosan. The obtained colloidal system showed long-term stability for about 6 months without any sign of aggregation. The particles are used as catalyst for the reduction of eosin in aqueous solution, which was monitored by means of spectrofluorometry and spectrophotometry. In order to identify the mechanism of reduction of dye with AuNPs, fluorescence lifetime studies were conducted. The result obtained from these studies ruled out the possibility of quenching of fluorescence of dye in presence of AuNPs and confirmed that the decrease in intensity on the absorption and emission spectra is mainly attributed to the reduction of dye catalyzed by AuNPs. In the light of these results, chitosan-capped AuNPs could be considered as an efficient nanocatalyst for different organic reactions.

\section{Additional files}

Additional file 1: UV-Vis spectra showing the effect of microwave irradiation time on the formation of AuNPs. a) $30 \mathrm{~s}$, b) $60 \mathrm{~s}, \mathrm{c}) 90 \mathrm{~s}, \mathrm{~d}$ ) $120 \mathrm{~s}, 150 \mathrm{~s}$ [ HAuCl4-0.005 M, chitosan-50 mg, microwave power-600W ].

Additional file 2: UV-Vis spectra of AuNPs synthesized using different chitosan concentrations. a) $10 \mathrm{mg}$, b) $20 \mathrm{mg}$, c) $30 \mathrm{mg}$, d) 40 mg, e) 50 mg, f) 60 mg, g) 70 mg [ HAuCl4-0.005 M, microwave irradiation time-60 s, power-600W ].

\section{Competing interests}

The authors declare that they have no competing interest.

\section{Authors' contributions}

All authors provided the same contributions in this article. All authors read and approved the final manuscript.

\section{Acknowledgements}

We are very grateful to the professor and head of the Department of Chemistry, University of Kerala for the facilities and support. The authors wish to thank NIIST, Trivandrum for carrying out the TCSPC measurements.

Received: 20 June 2012 Accepted: 25 September 2012

Published: 17 October 2012

\section{References}

1. Astruc, D, Lu, F, Aranzaes, JR: Nanoparticles as recyclable catalysts: the frontier between homogeneous and heterogeneous catalysis. Angew. Chem. Int. Ed. 44(48), 7852-7872 (2005)

2. Panyala, NR, Pena-Mendez, EM, Havel, J: Gold and nano-gold in medicine: overview, toxicology and perspectives. J. Appl. Biomed. 7(2), 75-91 (2009)

3. Grunwaldt, JD, Kiener, C, Wögerbauer, C, Baiker, A: Preparation of supported gold catalysts for low-temperature CO oxidation via "size-controlled" gold colloids. J. Catal. 181, 223-227 (1999) 
4. Carnes, CL, Klabunde, KJ: Synthesis, isolation, and chemical reactivity studies of nanocrystalline zinc oxide. Langmuir 16, 3764-3772 (2000)

5. Pal, T, Jana, NR, Sau, TK: Nucleophile induced dissolution of gold. Corros. Sci. 39, 981-985 (1997)

6. Grace, AN, Pandian, K: One pot synthesis of polymer protected gold nanoparticles and nanoprisms in glycerol. Colloids Surf A 290, 138-142 (2006)

7. Wangoo, N, Bhasin, KK, Mehta, SK, Suri, CR: Synthesis and capping of waterdispersed gold nanoparticles by an amino acid: bioconjugation and binding studies. J. Colloids Interface Sci. 323, 247-254 (2008)

8. He, S, Guo, Z, Zhang, Y, Zhang, S, Wang, J, Gu, N: Biosynthesis of gold nanoparticles using the bacteria Rhodopseudomonas capsulate. Mater. Lett. 61, 3984-3987 (2007)

9. Wei, DW, Qian, WP, Shi, Y, Ding, SH, Xia, Y: Effects of cooling treatment and glutaraldehyde on the morphology of Au nanostructures synthesized from chitosan. Carbohydr. Res. 343, 512-620 (2008)

10. Raveendran, P, Fu, J, Wallen, SL: Completely "green" synthesis and stabilization of metal nanoparticles. J. Am. Chem. Soc. 125, 13940-13943 (2003)

11. Mukherjee, P, Ahmad, A, Mandal, D, Senapati, S, Sainkar, SR, Khan MI Parishcha, R, Ajaykumar, PV, Alam, M, Kumar, R, Sastry, M: Fungus-mediated synthesis of silver nanoparticles and their immobilization in the mycelial matrix: a novel biological approach to nanoparticle synthesis. Nano Lett 1(10), 515-1519 (2001)

12. Mandal, S, Selvakannan, PR, Phadiare, S, Pasricha, R, Sastry, M: Synthesis of a stable gold hydrosol by the reduction of chloroaurate ions by the amino acid, aspartic acid. Proc. Indian Acad. Sci. (Chem. Sci.) 114, 513-520 (2002)

13. Selvakannan, PR, Anita, S, Srisathya, ND, Shirude, S, Pasricha, R, Mandale, A, Sastry, M: Synthesis of aqueous Au core-Ag shell nanoparticles using tyrosine as a pH-dependent reducing agent and assembling phasetransferred silver nanoparticles at the air-water interface. Langmuir 20, 7825 (2004)

14. Wei, DW, Qian, WP, Shi, Y, Ding, SH, Xia, Y: Mass synthesis of single-crystal gold nanosheets based on chitosan. Carbohydr. Res. 342, 2494-2499 (2007)

15. Wei, DW, Qian, WP: Facile synthesis of Ag and Au nanoparticles utilizing chitosan as a mediator agent. Colloids Surf. B. 62, 136-142 (2008)

16. Ishizuki, KE, Kanjiro, T, Kenjiro, M: Characterization of precious metal particles prepared using chitosan as a protective agent. Colloids Surf. 55, 15-18 (1991)

17. Liu, Y, Peng, C, Linyong, S, Fang, Y: Synthesis of silver nanoparticles by $Y$-ray irradiation in acetic water solution containing chitosan. Radiat. Phys. Chem. 76, 1165-1169 (2007)

18. Yang, $H$, Shen, $G, Y u, R$, Yang, M, Yang, Y: Layer-by-layer self assembled multilayer films of carbon nanotubes and platinum nanoparticles with polyelectrolyte for the fabrication of biosensors. Biomaterials 27, 246-255 (2006)

19. Yang, X, Huang, H: Synthesis of chitosan-stabilized gold nanoparticles in the absence/presence of tripolyphosphate. Biomacromolecules 5 , 2340-2346 (2004)

20. Raveendran, P, Fu, J, Wallen, SL: A simple and "green" method for the synthesis of $\mathrm{Au}, \mathrm{Ag}$, and $\mathrm{Au}-\mathrm{Ag}$ alloy nanoparticles. Green Chem. 8, 34-38 (2006)

21. Liu, J, Qin, G, Raveendran, P, Ikushima, Y: Facile "green" synthesis, characterization, and catalytic function of $\beta$-D-glucose-stabilized Au nanocrystals. Chem. Eur. J. 12, 2131-2138 (2006)

22. Huang, $H$, Yang, $X$ : Synthesis of polysaccharide-stabilized gold and silver nanoparticles: a green method. Carbohydr. Res. 339, 2627-2631 (2004)

23. Yamauchi, Y, Takai, A, Nagaura, T, Inoue, S, Kuroda, K: Pt Fibers with stacked donut-like mesospace by assembling Pt nanoparticles: guided deposition in physically confined self-assembly of surfactants. J. Am. Chem. Soc. $130,5426-5427$ (2008)

24. Mao, X, Ma, Y, Zhang, A, Zeng, A, Liu, G: Disposable nucleic acid biosensors based on gold nanoparticle probes and lateral flow strip. Anal. Chem. 81, 1660-1665 (2009)

25. Tanahashi, I, Manabe, Y, Tohda, T, Sasaki, SA, Nakamura, A: Optical nonlinearities of $\mathrm{Au} / \mathrm{SiO}_{2}$ composite thin films prepared by a sputtering method. J. Appl. Phys. 79, 1244-1245 (1996)

26. Wei, D, Sun, W, Qian, W, Ye, Y, Mac, X: The synthesis of chitosan-based silver nanoparticles and their antibacterial activity. Carbohydr. Res. 344 2375-2378 (2009)
27. Huang, L, Zhai, M, Peng, J, Xu, L, Li, J, Wei, GJ: Synthesis, size control and fluorescence studies of gold nanoparticles in carboxymethylated chitosan aqueous solutions. J Colloid Interface Sci. 316, 398-404 (2007)

28. Chandran, SP, Chaudhary, MR, Pasricha Ahmad, A, Sastry, M: Synthesis of gold nanotriangles and silver nanoparticles using aloe vera plant extract. Biotechnol. Prog. 22, 577-583 (2006)

29. Panigrahi, S, Basu, S, Praharaj, S, Pande, S, Jana, S, Pal, A, Ghosh, SK, Pal, T: Synthesis and size-selective catalysis by supported gold nanoparticles: study on heterogeneous and homogeneous catalytic process. J. Phys. Chem. C $111,4596-4605$ (2007)

30. Tsuji, M, Hashimoto, M, Nishizawa, Y, Tsuji, T: Synthesis of gold nanorods and nanowires by a microwave-polyol method. Mater. Lett. 58, 2326-2328 (2004)

31. Li, CC, Cai, WP, Cao, BQ, Sun, FQ, Li, Y, Kan, CX, Zhang, LD: Synthesis of hexagonal-phase NaYF4:Yb, Er and NaYF4:Yb, Tm nanocrystals with efficient up-conversion fluorescence. Adv Funct Mater 16, 2324-2329 (2006)

32. Frelink, T, Visscher, W, Veen, J: Particle size effect of carbon-supported platinum catalysts for the electrooxidation of methanol. J. Electroanal. Chem. 382, 65-72 (1995)

33. Ghosh, SK, Kundu, S, Mandal, M, Pal, A: Silver and gold nanocluster catalyzed reduction of methylene blue by arsine in a micellar medium. Langmuir 18, 8756-8760 (2002)

34. Bond, GC: Gold: a relatively new catalyst. Catal. Today 72, 5-9 (2002)

35. Pelet, S, Gratzel, M, Moser, JE: Femtosecond dynamics of interfacial and intermolecular electron transfer at eosin-sensitized metal oxide nanoparticles. J. Phys. Chem. B 107, 3215-3224 (2003)

36. Ghosh, SK, Mandal, M, Kundu, S, Nath, S, Pal, T: Bimetallic Pt-Ni nanoparticles can catalyze reduction of aromatic nitro compounds by sodium borohydride in aqueous solution. Appl Catal A 61, 268-2674 (2004)

37. Jana, S, Pande, S, Panigrahi, S, Praharaj, S, Basu, S, Pal, A, Pal, T: Exploitation of electrostatic field force for immobilization and catalytic reduction of o nitrobenzoic acid to anthranilic acid on resin-bound silver nanocomposites. Langmuir 22, 7091-7095 (2006)

38. Fukuoka, A, Higashimoto, N, Sakamoto, Y, Inagaki, S, Fukushima, Y, Ichikawa, M: Ship-in-bottle synthesis and catalytic performances of platinum carbonyl clusters, nanowires, and nanoparticles in micro- and mesoporous materials. Catal Today 66, 23-31 (2002)

39. Berezin, MY, Achilefu, S: Fluorescence lifetime measurements and biological imaging. Chem. Rev. 110, 2641-2684 (2010)

doi:10.1186/2228-5326-2-26

Cite this article as: Komalam et al:: Designed plasmonic nanocatalysts for the reduction of eosin Y: absorption and fluorescence study. International Nano Letters 2012 2:26.

\section{Submit your manuscript to a SpringerOpen ${ }^{\odot}$ journal and benefit from:}

- Convenient online submission

- Rigorous peer review

- Immediate publication on acceptance

- Open access: articles freely available online

- High visibility within the field

- Retaining the copyright to your article

Submit your next manuscript at $\gg$ springeropen.com 